\title{
Citações nos artigos publicados na Revista Portuguesa de Clínica Geral: análise da década 2000-2009
}

Maria da Luz Antunes*

\section{RESUMO}

Objectivos: Analisar as citações dos artigos publicados na Revista Portuguesa de Clínica Geral (RPCG) desde 2000, quando passou a ser disponibilizada online gratuitamente, aumentando a divulgação dos conteúdos da revista e alterando o seu impacto na comunidade científica.

Tipo de Estudo: Observacional, transversal e descritivo.

Local: Publicação online da RPCG.

População: Citações publicadas entre 2000 e 2009.

Métodos: Análise de citações dos artigos publicados na RPCG entre 2000 e 2009, contabilizando o total de citações, sua tipologia, os periódicos internacionais e nacionais mais citados e identificando as bases de dados mais citadas.

Resultados: Aumento do número de citações a partir de 2006. Os artigos das revistas científicas foram o documento privilegiado para a divulgação de resultados científicos com $66,27 \%$. A RPCG foi a revista mais citada. Três das revistas mais citadas constam da lista das 20 mais citadas quando analisado o factor de impacto. As revistas mais citadas não são de acesso livre e são assinadas por muitas bibliotecas portuguesas da área da saúde. Das citações das bases de dados, 83,3\% são feitas a partir de 2005.

Conclusões: Os autores dos artigos privilegiaram os artigos científicos para a divulgação dos resultados da investigação, em detrimento de comunicações em congressos ou em livros. Nas revistas mais citadas são identificados três títulos (New England Journal of Medicine, Lancet e JAMA) posicionados no ranking de análise do factor de impacto junto da comunidade científica. O presente estudo aponta também uma clara preferência dos autores pela citação da própria RPCG, a que não será alheio o seu acesso online, e para uma crescente procura de bases de dados de conteúdos temáticos bem definidos: revisão sistemática e medicina baseada na evidência.

Palavras-chave: Bibliometria; Bibliografia como assunto.

\section{INTRODUÇÃO}

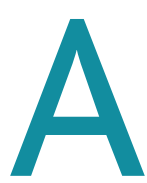
s revistas científicas representam o principal canal de comunicação dos investigadores para dar a conhecer o resultado do seu trabalho. ${ }^{1}$ Ainda que desigual entre as diferentes áreas do conhecimento, o artigo científico constitui a tipologia documental preferida, ${ }^{2}$ sendo que, na área das Ciências da Saúde, mais de $90 \%$ dos investigadores ${ }^{3}$ privilegia o artigo científico como a melhor forma de di-

* Mestre em Ciências Documentais. Coordenadora da Biblioteca da Escola Superior de Tecnologia da Saúde de Lisboa (ESTeSL) - Instituto Politécnico de Lisboa. vulgar o seu trabalho.

Deste modo, a análise de citações de revistas científicas permite identificar conceitos de uma especialidade, conhecer as tendências da investigação, bem como a literatura utilizada numa determinada disciplina. São, então, frequentes os estudos bibliométricos de análise de citações em revistas científicas, já que permitem conhecer quantitativamente alguns dos seus indicadores bibliométricos: 1) quantificar a importância e o impacto de autores, departamentos, instituições, países e investigações científicas; ${ }^{4}$ 2) quantificar a importância de revistas científicas através da sua referenciação e da 
sua quantificação em plataformas científicas, como a Web of Knowledge, o SCOPUS ou o SCImago Journal Rank. Esta quantificação pode ser analisada, entre outros elementos, por ano, pelo número de investigadores envolvidos nas equipas e também pelos recursos financeiros envolvidos (em regime de bolsas de investigação, por exemplo).

São conhecidos exemplos em que os estudos bibliométricos têm directa intervenção na gestão de serviços de saúde: ${ }^{4}$ por exemplo, parte da percentagem do financiamento dos hospitais pelo governo finlandês depende do factor de impacto das revistas onde os profissionais de saúde do hospital publicam os seus artigos; e, na Alemanha, as verbas a distribuir aos investigadores universitários são apuradas com base numa fórmula que inclui o Factor de Impacto da ISI Thomson Scientific. $^{5}$

Deste modo, a bibliometria surge como uma disciplina com um alcance multidisciplinar, que estuda a organização de sectores científicos e tecnológicos a partir das fontes bibliográficas, de modo a identificar actores, suas relações e suas tendências ${ }^{6}$ e cujas técnicas bibliométricas permitem identificar as tendências e o crescimento do conhecimento, as revistas científicas de uma disciplina, prever as tendências de uma publicação e também analisar os processos de citação. ${ }^{7}$

Para o médico de família é necessária uma actualização regular e satisfatória em todos os campos da Medicina e também em aspectos epidemiológicos, sociológicos e antropológicos específicos da Medicina Geral e Familiar (MGF). ${ }^{8}$ Em Portugal, a Revista Portuguesa de Clínica Geral (RPCG) é uma das revistas mais prestigiadas na especialidade da MGF. ${ }^{9}$ Mais: é considerada como a publicação de referência da MGF em Portugal, enquanto instrumento de formação e espaço de reflexão sobre a profissão, possui uma norma editorial bem definida e um sistema de revisão inter-pares dos artigos publicados, bem como uma boa acessibilidade dos seus conteúdos. ${ }^{10}$ É regularmente publicada desde 1984 sob a égide da Associação Portuguesa de Médicos de Clínica Geral, publica 6 números por ano e recebeu o ISSN 0870-7103. Está, desde Janeiro de 2011, indexada na plataforma SciELOPortugal (http://www.scielo.oces. mctes.pt/scielo.php/script_sci_serial/pid_08707103/lng_pt/nrm_iso). Ainda: desde o ano 2000 que a RPCG passou a ser disponibilizada online (em regime de open access) e de forma gratuita através do endereço: http://www.apmcg.pt/PageGen.aspx?WMCM_Paginald $=32651$.

Foi já objecto de um estudo bibliométrico, ${ }^{9}$ publicado em 2008, sob a forma de uma revisão sistemática dos números da RPCG publicados entre 2000 e 2006 quanto à tipologia de artigos, número total de referências bibliográficas, número de referências bibliográficas de artigos publicados em Portugal e número de referências bibliográficas de artigos já publicados na própria RPCG nos anos em análise. Também o peso da citação de artigos foi o principal objectivo deste estudo bibliométrico.

Mais recentemente, já em 2010, a RPCG foi outra vez objecto de mais um estudo bibliométrico, desta vez envolvendo uma análise comparativa entre o número de citações de artigos espanhóis na RPCG e o número de citações de artigos portugueses na Atención Primaria. ${ }^{11}$ Assim, dado que a análise de citações num periódico constitui uma importante base de reflexão dos seus conteúdos, ${ }^{12}$ o objectivo do presente estudo é analisar as citações dos artigos publicados na RPCG entre os anos 2000 e 2009, altura em que passou a ser disponibilizada online em regime de open access-seguramente aumentando a divulgação dos conteúdos da revista e alterando o seu impacto na comunidade científica-, ao mesmo tempo que se pretende identificar o tipo de documentos citados, as revistas nacionais e internacionais mais citadas, bem como as bases de dados mais citadas.

\section{MÉTODOS}

Amostra: Citações dos artigos publicados na RPCG a partir do site da revista. Foi seleccionada a década de 2000 a 2009, inclusive; a partir de 2000 porque é a partir desta data que os conteúdos da revista são disponibilizados em acesso aberto e, até 2009, de molde a completar uma década de análise de citações.

Metodologia: Estudo observacional, transversal e descritivo da análise de citações de todos os artigos da RPCG publicados na década em estudo.

Procedeu-se à definição dos indicadores bibliométricos em análise: número total de citações, a tipologia das citações, as revistas internacionais e nacionais mais citadas e a identificação das bases de dados mais citadas. Para o levantamento de dados foram analisadas to- 


\section{QUADRO I. Total de citações (2000-2009)}

\begin{tabular}{cc} 
Ano & Total de citações \\
\hline 2000 & 748 \\
2001 & 452 \\
2002 & 547 \\
2003 & 776 \\
2004 & 875 \\
2005 & 880 \\
2006 & 1560 \\
2007 & 1491 \\
2008 & 1327 \\
2009 & 1365 \\
\hline TOTAL & 10021 \\
\hline
\end{tabular}

QUADRO II. Tipologia de documentos citada

\begin{tabular}{lrr} 
Tipologia & Citações & $\%$ \\
\hline Artigos & 6641 & 66,27 \\
Livros & 1741 & 17,37 \\
Capítulos & 635 & 6,33 \\
eDocuments & 474 & 4,73 \\
URLs & 174 & 1,73 \\
Legislação & 74 & 0,73 \\
Bases de Dados & 66 & 0,65 \\
Comunicações & 59 & 0,58 \\
Teses & 58 & 0,57 \\
Circulares & 37 & 0,36 \\
Software & 30 & 0,29 \\
Comunicações Pessoais & 12 & 0,11 \\
Forthcoming & 11 & 0,10 \\
Documentos de Formação & 7 & 0,06 \\
Audiovisuais & 2 & 0,01 \\
\hline TOTAL & 10021 & 100 \\
\hline
\end{tabular}

das as referências bibliográficas apresentadas em todos os artigos publicados e criada uma grelha de dados em Excel ${ }^{\circledR}$, onde foi registado o número de referências por número e por ano de publicação, bem como todos os dados referentes aos vários tipos de documentos citados pelos autores. Esta grelha foi alargada sempre que um novo tipo de documento era citado (e.g., circulares ou comunicações pessoais).

Foram registados todos os títulos de revista, nacionais e internacionais, citados nos vários artigos e anotado o número de vezes que eram citados. Este registo permitiu identificar as revistas que eram mais e as que eram menos citadas. Registou-se, finalmente, a frequência de citações das bases de dados citadas pelos autores, de modo a indagar da sua importância para a pesquisa de informação.

\section{RESULTADOS}

No presente estudo, em dez anos de publicação, verifica-se que a RPCG publicou 597 artigos, os quais apresentam um total de 10.021 citações (Quadro I) - em média, cada artigo citou 16,78 referências bibliográficas. De assinalar o aumento de citações a partir de 2006.

No Quadro II é identificada a tipologia de documentos citada nas referências bibliográficas dos artigos da RPCG. Assinale-se o número de artigos, de livros, de documentos electrónicos e o leque de documentos considerados: desde legislação a documentos em vias de publicação (forthcoming) e a comunicações pessoais, decorrentes habitualmente de conversas com os respectivos autores.

Foi citado um total de 1.323 diferentes títulos de revistas. O Quadro III apresenta a lista das 20 revistas, nacionais e internacionais, mais citadas na RPCG. O Quadro IV identifica um conjunto restrito de títulos de revistas que, isoladas, atingem mais de $25 \%$ das citações. Considerando o número total de revistas citado (1.323), assinale-se o número de revistas com poucas citações (QuadroV), em especial as que são citadas apenas uma ou duas vezes.

Relativamente às revistas nacionais, o Quadro VI apresenta a lista das 20 mais citadas na RPCG. Registaram-se 967 citações para um total de 124 diferentes títulos de revistas. As citações das revistas nacionais representam $6,86 \%$ do total, enquanto as revistas nacionais representam 10,66\% do total de títulos citados.

$O$ recurso a bases de dados internacionais pode ser mais uma opção na pesquisa de informação. Neste caso, o Quadro VII identifica as preferências dos investigadores pelas bases de dados.

\section{CONCLUSÕES}

Sendo uma das mais importantes revistas de refe- 


\begin{tabular}{|rlr|}
\hline \multicolumn{2}{|l}{ QUADRO Ill. Lista das 20 revistas, nacionais e } \\
internacionais, mais citadas \\
\hline N. & Título & Citações \\
\hline 1 & Revista Portuguesa de Clínica Geral & 507 \\
2 & BMJ & 342 \\
3 & JAMA & 210 \\
4 & Lancet & 176 \\
5 & New England Journal of Medicine & 169 \\
6 & Diabetes Care & 121 \\
7 & American Family Physician & 98 \\
8 & Pediatrics & 81 \\
9 & Atención Primaria & 79 \\
10 & Journal of Family Practice & 77 \\
11 & British Journal of General Practice & 76 \\
12 & Circulation & 75 \\
13 & Annals of Internal Medicine & 68 \\
14 & Archives of Internal Medicine & 64 \\
15 & Family Practice & 60 \\
16 & Canadian Medical Association Journal & 50 \\
17 & Acta Médica Portuguesa & 45 \\
18 & Canadian Family Physician & 40 \\
19 & American Journal of Medicine & \\
20 & Social Science and Medicine & \\
& & 61 \\
\hline
\end{tabular}

rência para a MGF e analisadas as citações na RPCG, constata-se que a análise cruzada das citações indirectamente se assumiu como um indicador de impacto e de estilo da revista.

De assinalar o aumento de citações nos artigos publicados a partir de 2006, altura em que o acesso ao portal da $b$-on é alargado a todas as instituições públicas de ensino superior, a laboratórios estatais de investigação e a um conjunto de sete hospitais públicos. Desta forma, o acesso à informação médica está, ou ficou, nos últimos anos, cada vez mais facilitado, ${ }^{13}$ ainda

\begin{tabular}{|rr|}
\hline QUADRO V. Número de revistas com menos citações \\
\hline N. ${ }^{\circ}$ de Citações & N. $^{\circ}$ de Revistas \\
\hline 9 & 14 \\
8 & 14 \\
7 & 24 \\
6 & 35 \\
5 & 42 \\
4 & 60 \\
3 & 106 \\
2 & 235 \\
1 & 675 \\
\hline
\end{tabular}

que de um modo geral inacessível a partir dos centros de saúde onde justamente exerce a maioria dos autores que publica na RPCG. Registe-se que o acesso ao texto integral de publicações científicas é, nos tempos que correm, cada vez mais fácil e em número significativo, seja porque cada vez são editadas mais revistas na área da saúde, seja porque algumas publicações estão disponíveis gratuitamente em regime de open access (em acesso imediato, como as dos repositórios digitais da Biomed Central e da Pubmed Central, por exemplo, ou após um período de espera que pode ir de alguns meses até dois ou três anos), seja porque este acesso é garantido por assinaturas em consórcio. ${ }^{13}$ Não podemos, porém, ignorar que o acesso de médicos de família e de internos de MGF a conteúdos pagos é muito assimétrico a nível nacional (mais limitado no Sul) e que é

\begin{tabular}{|clrrr|}
\hline \multicolumn{2}{|c}{ QUADRO IV. Revistas que contemplam mais de $25 \%$ das citações. } & \\
\hline N. ${ }^{\circ}$ & Título & Citações & $\%$ & Freq. Acum. \\
\hline 1 & Revista Portuguesa de Clínica Geral & 507 & 7,67 & (\%) \\
2 & BMJ & 342 & 5,17 & 12,85 \\
3 & JAMA & 210 & 3,17 & 16,03 \\
4 & Lancet & 176 & 2,66 & 18,70 \\
5 & New England Journal of Medicine & 169 & 2,55 & 21,25 \\
6 & Diabetes Care & 121 & 1,83 & 23,09 \\
7 & American Family Physician & 98 & 1,48 & 24,57 \\
8 & Pediatrics & 81 & 1,22 & 25,80 \\
\hline
\end{tabular}


QUADRO VI. Lista das 20 revistas portuguesas mais citadas

\begin{tabular}{|c|c|c|}
\hline N. ${ }^{\circ}$ & Título & Citações \\
\hline 1 & Revista Portuguesa de Clínica Geral & 507 \\
\hline 2 & Acta Médica Portuguesa & 45 \\
\hline 3 & Revista Portuguesa de Saúde Pública & 33 \\
\hline 4 & Arquivos de Medicina & 23 \\
\hline 5 & Saúde Infantil & 20 \\
\hline 6 & Acta Pediátrica Portuguesa & 16 \\
\hline 7 & Médico de Família & 16 \\
\hline 8 & Acta Pediátrica & 14 \\
\hline 9 & Revista Portuguesa de Cardiologia & 13 \\
\hline 10 & Revista Portuguesa de Pneumologia & 13 \\
\hline 11 & Diabetologia & 11 \\
\hline 12 & Nascer e Crescer & 10 \\
\hline 13 & Epilepsia & 8 \\
\hline 14 & Pediatria Integral & 8 \\
\hline 15 & Revista Portuguesa de Doenças Infecciosas & 8 \\
\hline 16 & Saúde em Números & 8 \\
\hline 17 & Boletim de Farmacovigilância & 6 \\
\hline 18 & Educação Médica & 6 \\
\hline 19 & Psiquiatria Clínica & 6 \\
\hline 20 & Teste Saúde & 6 \\
\hline
\end{tabular}

muitas vezes a participação em listas e fóruns de discussão que desencadeia a partilha de informação.

A crescente importância das citações a recursos electrónicos (numa associação de sites e de documentos electrónicos) pode ser explicada pelo auge da Internet na última década, que alterou de forma radical os processos de comunicação e de acesso à informação, e pelo facto de algumas publicações hoje estarem disponíveis exclusivamente em formato digital. Num estudo de 2009, médicos de família portugueses afirmam que a Internet é uma fonte muito importante para melhorar os seus conhecimentos, ${ }^{14}$ reforçando que os seus hábitos de leitura e pesquisa através da Internet aumentaram.

Por outro lado, também os artigos das revistas científicas publicados na RPCG assumem um importante papel no processo de institucionalização da própria MGF e são, para os seus autores, o tipo de documento privilegiado para a divulgação de resultados científi- $\cos ,{ }^{1-2,10}$ em detrimento de comunicações em congressos ou em livros, as quais poderão ser menos dinâmicas na difusão do conhecimento científico. ${ }^{2}$ Com $66,27 \%$ estão, porém, aquém dos $90 \%$ referidos noutro estudo. ${ }^{3}$

Nas revistas mais citadas, a nível internacional, identificam-se três títulos (New England Journal of Medicine, Lancet e JAMA) bem posicionados no ranking de análise do factor de impacto junto da comunidade científica - esta selecção aponta para a qualidade de conteúdos das mesmas e para uma tendência dos autores pela sua leitura. Sendo, porém, revistas não expressamente dirigidas aos cuidados de saúde primários e existindo revistas dirigidas aos cuidados de saúde primários e à MGF, poder-se-á apresentar a hipótese de a consulta preferencial destes títulos estar associada aos hábitos adquiridos durante a formação hospitalocêntrica da maioria dos médicos de família e internos de MGF.

Ainda no âmbito das revistas mais citadas, a nível internacional, constata-se que nenhuma é de acesso livre (Quadro IV). Destas, verifica-se que a $B M J$ é regularmente recebida em 47 bibliotecas da área da saúde em Portugal, a JAMA é assinada em 45, a Lancet em 65 bibliotecas, a New England Journal of Medicine em 69, a Diabetes Care em 9 bibliotecas, a American Family Physician em 6 bibliotecas e a Pediatrics em 29 bibliotecas. ${ }^{15}$ Em qualquer destas revistas, a sua distribuição pelo território nacional é apreciável, permitindo, assim, um acesso mais vantajoso aos autores que publicam na RPCG.

Neste conjunto de revistas são apresentadas algumas que, por sua vez, constam, de igual modo, da lista das 20 mais citadas quando analisado o factor de impacto do ano de 2009 pela base de dados Journal Citation Reports [New England Journal of Medicine $\left(3^{\text {a }}\right.$, com um FI de 51,41), Lancet (13. ${ }^{a}$, com um FI de 29,44) e JAMA (20. ${ }^{\text {a }}$, com um FI de 27,75)], onde é analisado o factor de impacto de 7.387 revistas na comunidade científica. ${ }^{16}$ Ignora-se se os autores consideraram este ranking, mas esta selecção aponta para a qualidade de conteúdos das mesmas e para uma tendência dos autores pela sua leitura.

O presente estudo aponta também uma preferência dos autores pela citação da própria RPCG. Porque é a referência nacional dentro da $\mathrm{MGF}^{9,11}$ porque existem 


\begin{tabular}{|lr|}
\hline \multicolumn{2}{|l|}{ QUADRO VII. As bases de dados citadas } \\
Bases de Dados & N. ${ }^{\circ}$ de Citações \\
\hline Cochrane & 43 \\
UpToDate & 13 \\
Medscape & 1 \\
Porbase & 1 \\
B-ON & 1 \\
Clinical Evidence & 1 \\
Science Direct & 1 \\
Embase & 1 \\
Scopus & 1 \\
WoK & 1 \\
Pascal & 1 \\
CAB Abstracts & 1 \\
\hline TOTAL & 66 \\
\hline
\end{tabular}

poucas revistas nacionais de conteúdos médicos e também pelo factor linguístico, parece-nos uma opção sólida e reflexo da qualidade da própria RPCG - cerca de 2/3 destas citações figuram em artigos de investigação e de revisão. Por outro lado, é o resultado da disponibilização da revista online desde o ano 2000 e em acesso gratuito.

Apesar de o acesso online ser uma condição facilitadora, quando ponderada a mesma condição facilitadora junto das revistas brasileiras ou espanholas, justamente pela sua proximidade linguística, ela é inexistente. Não encontramos nenhuma revista brasileira no conjunto das 20 internacionais mais citadas - também não figura qualquer revista brasileira quando considerado o conjunto das primeiras 100 . Relativamente às revistas espanholas, identificamos uma nas primeiras 20: a Atención Primaria. Quando considerado o conjunto das 100 primeiras, localizamos ainda Medicina Clinica (56. $\left.{ }^{\mathrm{a}}\right)$, Cadernos de Atención Primaria $\left(80 .^{\mathrm{a}}\right)$, Gaceta Sanitaria (91..$^{\mathrm{a}}$ e Anales Españoles de Pediatría (98. $\left.{ }^{\mathrm{a}}\right)$. Tal resultado corrobora a ideia da existência de uma ignorância mútua entre Portugal e Espanha em termos de produção científica ${ }^{11}$ e também a ideia de que parece que Espanha poderá ser mais importante para Portugal do que o contrário ${ }^{11}$ - num estudo, ${ }^{11}$ em 12 números publicados, a RPCG citava 3,1\% de referências es- panholas enquanto a Atención Primaria não citava uma única da RPCG.

Na investigação agora em curso e no âmbito das revistas nacionais mais citadas, verifica-se que a Acta Médica Portuguesa (indexada na Excerpta Médica, Índex Medicus, Chemical Abstracts, MEDLINE, Índex Copernicus, Directory of Open Access Journals, Índex RMP e Web of Science) é regularmente recebida em 69 bibliotecas portuguesas da área da saúde, que a Revista Portuguesa de Saúde Pública (disponibilizada no site e na plataforma SciELO) é assinada por 108 bibliotecas, que os Arquivos de Medicina (indexados na EMBASE/Excerpta Médica, na Latindex e no Índex RMP e disponíveis também na plataforma SciELO) são assinados por 38 bibliotecas e que a Saúde Infantilé regularmente recebida em 59 bibliotecas. ${ }^{15}$ Também aqui, a distribuição destas revistas pelo território nacional é vantajosa para leitores e potenciais autores na RPCG.

Curiosamente, as revistas BMJ, JAMA, Lancet e New England Journal of Medicine que, conjuntamente com a RPCG, compõem os primeiros cinco títulos mais citados na lista geral (Quadro III), não são de acesso livre e, em alguns casos, têm um considerável período de embargo para a disponibilização dos seus conteúdos. Estes resultados não estão completamente de acordo com os demonstrados num outro estudo, ${ }^{14}$ em que as três fontes científicas mais importantes de informação para os médicos de família portugueses eram a RPCG (confere), o Jornal Médico de Família e a American Family Physician - fontes de informação, decerto, mas não as principais fontes de citação no presente estudo. Mas, quando estudadas as cinco revistas médicas mais conhecidas e reputadas pela sua qualidade num outro estudo, ${ }^{10}$ a mais referida foi a RPCG, indicada por $70 \%$ de internos e orientadores do internato de MGF - dado que se confirma. Confirma-se, mas não com a mesma expressividade.

Constata-se o alargado número (1.323) de revistas citadas nos artigos da RPCG. Mas constata-se também um elevado número de revistas, de temas variados, com poucas citações (Quadro V). Porque foram então citadas? Atrevemo-nos a encontrar a resposta justamente no vasto leque de informação a que os profissionais de MGF devem aceder, actualizar-se, procurar respostas e soluções. Não cremos que exista outra especialidade médica que abarque tal volume de informação a considerar 
e controlar. Mas não podemos ignorar o estudo em que se consideram insuficientes os conteúdos das revistas médicas publicadas actualmente em Portugal para a satisfação das necessidades de leitura dos médicos. ${ }^{10}$

Aponte-se, ainda, a crescente procura de bases de dados de conteúdos temáticos bem definidos a partir de 2005: 83,3\% das citações são posteriores ao ano de 2005. Denota-se alguma confusão entre bases de dados, plataformas e portais, dado que todos estes recursos foram, nas referências bibliográficas, identificados como bases de dados; desde que devidamente citados e referenciados, uma designação menos clara é um problema menor. Na análise de citações do presente estudo, existe um predomínio pelos conteúdos de revisão sistemática (Cochrane Database of Systematic Reviews) e de medicina baseada na evidência (UpToDate). Tratase, em ambos os casos, de conteúdos pagos, mas, ainda assim, tudo aponta para uma preferência por recursos electrónicos temáticos e que apresentam a informação de forma sistematizada. Dada a sua procura nos anos mais recentes, deverá a sua assinatura ser ponderada pelas instituições de ensino superior da área da saúde ou a sua inclusão em consórcios como a b-on, em especial no pacote das bibliotecas hospitalares, ou criar um pacote especial a disponibilizar aos profissionais dos centros de saúde (à semelhança do que sucede na área da Administração Regional de Saúde do Norte).

Para concluir, refira-se que foram localizados alguns títulos de revistas assinados em numerosas bibliotecas ao longo do território nacional. São procuradas e seguramente lidas pelos seus leitores, mas, em tempos de constrangimentos financeiros, sugere-se uma política de assinaturas mais equilibrada, por parte dos gestores de Biblioteca, de molde a aumentar o leque de títulos disponível. A cada nova assinatura dever-se-á indagar da sua existência na área regional ou mesmo na cidade; qualquer gestor de Biblioteca o poderá fazer e tem os recursos para o fazer.

Cremos, como último apontamento, que a MGF, à semelhança de outras especialidades médicas e de outras ciências, continuará a desenvolver-se através dos trabalhos de investigação redigidos e publicados pelos seus profissionais. Ganha a MGF e ganham os mesmos profissionais que lamentam a falta de poucos conteúdos médicos em língua portuguesa editados em Portugal.

\section{REFERÊNCIAS BIBLIOGRÁFICAS}

1. Grol R, Zwaard A, Mokkink H, Dalhuijsen J, Casparie A. Dissemination of guidelines: which sources do physicians use in order to be informed? Int J Qual Health Care 1998 Apr; 10 (2): 135-40.

2. González Alcaide G, Alonso Arroyo A, Valderrama Zurián JC, Aleixandre Benavent R. Una década de investigaciones en Anales de Documentación (1998-2007): aproximación bibliométrica y temática. Ann Documentación 2008; 11: 57-78.

3. Bordons M, Gómez-Caridad I. La actividad científica española a través de indicadores bibliométricos en el período 1990-93. Rev Gen Información Documentación 1997;7 (2): 69-86.

4. Donato $\mathrm{H}$, Oliveira CF. Avaliação da produção científica portuguesa na área da ginecologia e obstetrícia baseada em indicadores bibliométricos. Acta Obstet Ginecol Port 2009; 3 (3): 107-14.

5. Wilcox AJ. Rise and fall of the Thomson Impact Factor. Epidemiology 2008 May; 19 (3): 373-4.

6. Spinak E. Diccionario enciclopédico de bibliometría, cienciometría e informetría. Caracas: UNESCO; 1996.

7. Vanti NA. Da bibliometria à webometria: uma exploração conceitual dos mecanismos utilizados para medir o registro da informação e a difusão do conhecimento. Ci Inf 2002 Mai-Ago; 31 (2): 152-62.

8. Nylenna M, Aasland OG. Primary care physicians and their information-seeking behaviour. Scand J Prim Health Care 2000 Mar; 18 (1): 9 13.

9. Rosendo I, Santos T, Martins D, Pimenta G, Neto MG, Francisco MP, et al. A citação da Revista Portuguesa de Clínica Geral na Revista Portuguesa de Clínica Geral. Rev Port Clin Geral 2008 Jul-Ago; 24 (4): 45761.

10. Sousa JC, Mateus A. Revistas médicas e formação em medicina geral e familiar: hábitos e necessidades de leitura de internos e de orientadores de formação do internato complementar. Rev Port Clin Geral 2005 JanFev; 21 (1): 23-42.

11. Villanueva T, Cavadas LF, Gérvas J, Padilla J, Serrano E, Cofiño R. Political borders as scientific barriers in general practice: Portugal and Spain as a case study. Eur J Gen Pract 2010 Sep; 16 (3): 151-3.

12. Nebelong-Bonnevie E, Frandsen TF. Journal citation identity and journal citation image: a portrait of the Journal of Documentation. J Documentation 2006; 62 (1): 30-57.

13. Montenegro M. Acessibilidade das publicações periódicas com maior interesse para a medicina geral e familiar. Rev Port Clin Geral 2006 JulAgo; 22 (4): 423-30.

14. Dieguez T, Teixeira A. A utilização das tecnologias de informação e comunicação pelos médicos de família portugueses: um estudo exploratório. Rev Port Clin Geral 2009 Mai-Jun; 25 (3): 281-304.

15. APDIS. APDIS Online [Internet]. Lisboa: APDIS; 2010 [cited 2010 Dec 27]. Disponível em: http://www.apdis.org [acedido em 27/12/2010].

16. Journal Citation Reports. Thomson Reuters; 2010. Disponível em: http://admin-apps.isiknowledge.com/JCR/JCR? PointOfEntry $=$ Home \&SID =V1kEnDMomGPCjMaji33 [acedido em 28/12/2010].

\section{CONFLITOS DE INTERESSE}

A autora declara não possuir qualquer tipo de conflito de interesses. 
ENDEREÇO PARA CORRESPONDÊNCIA

Maria da Luz Antunes

Av. D. João II, Lote 4.69 .01

1990-096 Lisboa
E-mail: mluz.antunes@estesl.ipl.pt; mluzcc@gmail.com

Recebido em 05/01/2011

Aceite para publicação em 04/05/2011

\section{ABSTRACT}

\section{CITATION ANALYSIS OF THE ARTICLES PUBLISHED IN REVISTA PORTUGUESA DE CLÍNICA GERAL BETWEEN 2000 AND 2009}

Objectives: To analyze the citations of the articles published in the Revista Portuguesa de Clínica Geral (RPCG) between 2000 and 2009 following the free publication of the journal online.

Type of study: Descriptive study.

Setting: RPCG online.

Unit of analysis: Citations of articles published between 2000 and 2009.

Methods: A citation analysis of all articles published in RPCG between 2000 and 2009 was conducted. Citations were counted, the typology of the citations was identified, the frequency of citation of international and national periodicals was noted, and the most frequently cited databases were recorded.

Results: The number of citations per article has increased since 2006. Articles from scientific publications were the source of most citations (66.27\%). RPCG was the journal cited most often. Three of the most cited periodicals are among the 20 journals with the highest impact factor (New England Journal of Medicine, Lancet and JAMA). The most cited periodicals do not provide free access but they are found in several health libraries in Portugal. Most citations of databases (83.3\%) are from after 2005.

Conclusions: The authors of the articles published in RPCG preferred citations to scientific articles rather than congress proceedings or books. Three journals with high impact factors were the most cited. This study also found a preference for citing the RPCG itself. This is probably linked to open online access. There was also an increased demand for databases with well defined contents for the preparation of systematic reviews and evidence-based medicine reviews.

Keywords: Bibliometrics; Bibliography as Topic. 